\title{
PA-119 EBOLA AND CLINICAL TRIAL ACTIVITY ON THE AFRICAN CONTINENT
}

Elizabeth Pienaar, Tamara Kredo. SACC, South Africa

10.1136/bmjgh-2016-000260.148

Background Ebola virus disease (EVD) results in an often fatal acute, serious illness. There are no effective EVD treatments, however, there is ongoing research into potential interventions in affected African countries. Since 2005, in efforts to enhance transparency researchers must register trials on one of World Health Organization's clinical trials registries. WHO's International Clinical Trial Registry Platform (ICTRP) collates this data providing information about planned, ongoing or 
completed trials for researchers, funders and the public. This study mapped African EVD trial activity as found on WHO's ICTRP and identified available evidence from trial publications.

Methods We conducted a cross-sectional analysis of EVD studies registered on ICTRP. Data extraction included trial location, intervention, participant age, and funders. We used registry identifiers to search PubMed for publications. Descriptive analysis was conducted in MS Excel'TM.

Results ICTRP was searched (20 June 2016) identifying 83 EVD studies. Of these 45 are Africa-based. Studies were registered from 2009-2016. Recruitment status indicates 6 completed, 2 withdrawn, 1 not started, 2 unknown status and 34 ongoing. Forty-one studies evaluate an intervention, 4 are observational. Interventions include vaccines (25), therapeutics (17), health services/care (1) and diagnostics (2). Children were included in 24 studies. Funding sources include local and international universities and governments, non-governmental organisations, and pharmaceutical industry. Of the 45 registered African studies, 11 records were found on PubMed, seven of which included results of EVD studies.

Conclusions Mapping EVD clinical trial activity on ICTRP and searching for completed studies on PubMed can provide data on planned, ongoing or completed trials. The current research focus is on identifying safe and efficacious vaccines to prevent EVD including in children. The low number of trial reports indicates that evidence is not yet publicly accessible which may impact on evidence-informed policy development for the region. 